\title{
IRST testing methodologies: Maritime Infrared Background Simulator
}

\author{
Piet B.W. Schwering
}

TNO Defence, Security and Safety, P.O. Box 96864, NL-2509 JG The Hague, Netherlands, piet.schwering@tno.nl

\begin{abstract}
In this paper we discuss methodologies to incorporate the effects of environments and scenarios in the testing of IRST systems. The proposed methodology is based on experience with sea based IRST trials combining the possibilities of performance assessment in required scenarios to the real performance in available coastal scenarios. For this purpose testing procedures depend strongly on accurate infrared target, background and atmosphere models. In particular, background effects can be dominating the performance in clutter conditions and can also dominate contrast values. For this purpose a maritime background model has been developed for use in test procedures. The model generates a scene image sequence containing background structure, including sea, sky, clouds, coastal and sun glint information up to a frame rate of $25 \mathrm{~Hz}$.
\end{abstract}

Keywords: Maritime background modeling, Infrared Search and Track, detection, system testing

\section{INTRODUCTION}

Infrared Search and Track (IRST) systems have been in development since the 1970's. On board naval vessels, large detection potential was gained in scenarios and situations where point like targets are present. Several IRST systems, such as VAMPIR and IRSCAN, have been introduced on operational vessels, and the next generation, such as SIRIUS ${ }^{1}$, is being introduced right now. These systems are primarily designed for (long range) point target detection, such as missiles. For future use several changes in the operational context will take place. First of all, other types of threats must be considered in different operational scenarios and environments. The threats are basically of the small-extended type, and can be located at shorter range to the ship in coastal environments. Besides the fact that the type of targets has changed, secondly, also the number and the methods for making a distinction between targets and backgrounds, will be very different. This complicates the overall system performance assessment. Obviously, performing trials on these targets, such as small boats, is not always feasible from the operational point of view. In order to derive accurate system performance metrics, much more emphasis is needed from support of target, atmospheric and background models. With accurate scene model simulations several scenarios can be tested. In order to determine accurate scene models, effort must be taken to validate these models in various scenarios. This paper presents the efforts that have been taken at TNO to assess the performance of new type of IRSTs to detect and classify small surface targets.

With present-day scenario modeling, based on scene generator developed in the 1990's accurate predictions are made for relatively large targets, such as large naval vessels and airplanes. We have integrated such an airplane target model as part of our IRSCENE scene generator ${ }^{2}$, and are using inputs from the first target model in analysis via EOSTAR ${ }^{3}$. The simulations are originally developed for relatively well defined open ocean scenarios. Future IRST systems are being designed for use in coastal environments, and detection of small (and small extended) targets. These new systems are probably of the staring type with frame update rates of up to $25-30 \mathrm{~Hz}$, while present-day IRST's are slowly scanning (around $1 \mathrm{~Hz}$ ). For this purpose more accurate target and background models are required, as well as the interaction

Infrared Technology and Applications XXXII, edited by Bjørn F. Andresen, Gabor F. Fulop, Paul R. Norton, Proc. of SPIE Vol. 6206, 620621, (2006) · 0277-786X/06/\$15 - doi: 10.1117/12.667990 
between target and background. In this paper we describe our approach to analyzing and modeling the scenario simulation modeling. The aim of the modeling is to assess system performance by extrapolating over different targets and environments.

In this paper we proceed with a description of methodologies on how to improve system modeling to incorporate new targets, and particularly new backgrounds. A description is given of present-day background modeling, especially the Maritime Infrared Background Simulator developed at TNO, and approaches of the way forward.

\section{BACKGROUND MODEL SET-UP}

The Maritime Infrared Background Simulator (MIBS) is based on maritime background studies in open ocean scenarios from ship based and air based platforms. In previous publications (Schwering, $1992^{4}$ and $1996^{5}$ ) knowledge on maritime backgrounds was deduced in order to derive at accurate modeling of maritime backgrounds for IRST evaluation. The primary objectives at the time were to have an image physically representing a true scene, while background intensity and spatial clutter levels are accurate at an image repetition rate of $1 \mathrm{~Hz}$. At this frame rate subsequent images are assumed uncorrelated. A description and (spatial intensity, clutter and white cap generation) validation of the algorithm is given in Schwering $\left(1996^{5}\right)$. Due to the complexity of background images it was chosen that to a large extend recorded infrared backgrounds are used in determining intensity levels as well as clutter. The design drivers of the modeling are the background invariance with distance, the structured sea surface, the temperature decrease with height in the air, and the specific interface with the horizon. The main area of use is with near-horizon objects, which also requires a clutter decrease near the horizon due a lower number of visible white caps. White caps are visible as long tails of the intensity distribution of patches of sea surfaces. An important factor in white cap clutter generation is that the image keeps its continuity in intensity.

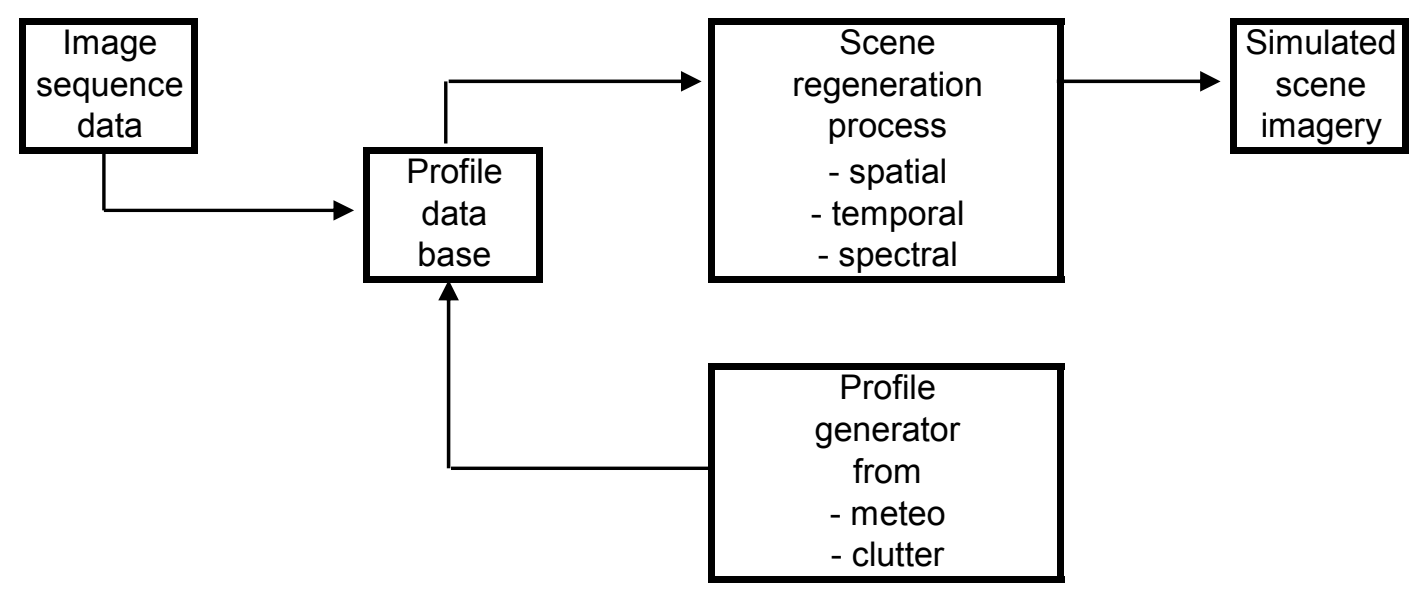

Figure 1: MIBS development and simulation process.

For the purpose of IRST testing, the maritime background model has been developed. The approach based on translation invariance of the background scene is maintained, as well as the use of an extensive database. The primary contributors to the received emission from backgrounds are surface emissions and reflections. Under grazing angles such as those in observing objects near the horizon from a ship board sensor, expected reflection coefficients are close to one, and hence for a flat surface reflection will dominate. For the sea surface two complications occur. First of all the surface is rippled and hence the excepted average slope is reflecting not from grazing angles but angles reflecting radiation from much higher elevation. Second, additional white caps will introduce foam and will result in an effect that the apparent 
temperature will become close to the true sea surface temperature. Therefore the expected emission from sea surface near the horizon is based on emissivity values ranging from close to zero to close to one. Due to decreased sensor spatial resolution at longer ranges an averaging effect needs to be taken into account as well. Note that these factors are very difficult to take into account in all detail, due to the size of the details compared to available data of the sea surface. Other long range effects, such as low transmission, and potentially strong scintillation, will lower the contrasts and blur the resolution.

The modeling process is given schematically in Figure 1. From real infrared imagery recorded in different scenarios data determining background vertical intensity profiles were derived, as well as clutter parameters such as local standard deviations and correlation coefficients in different locations in the scene (air, near horizon, sea). This led to a profile database of 124 different profiles. Input data to generate profile information was taken from NATO trials at SIVEX (1989), LAPTEX (1996), SIMVEX (2001), and national trials (WATEX and BALTIC, both 1999). These trials resulted in data in several different scenarios, warm-humid, cold, and Mediterranean. The profiles are obtained for the 3-5 and 812 micron bands, limiting simulations to sub-bands of MWIR and LWIR. As it was recognized that a wide variety of backgrounds occur, full control of background simulation seems appropriate, as well as a firm basis of the model on large datasets and image analysis.
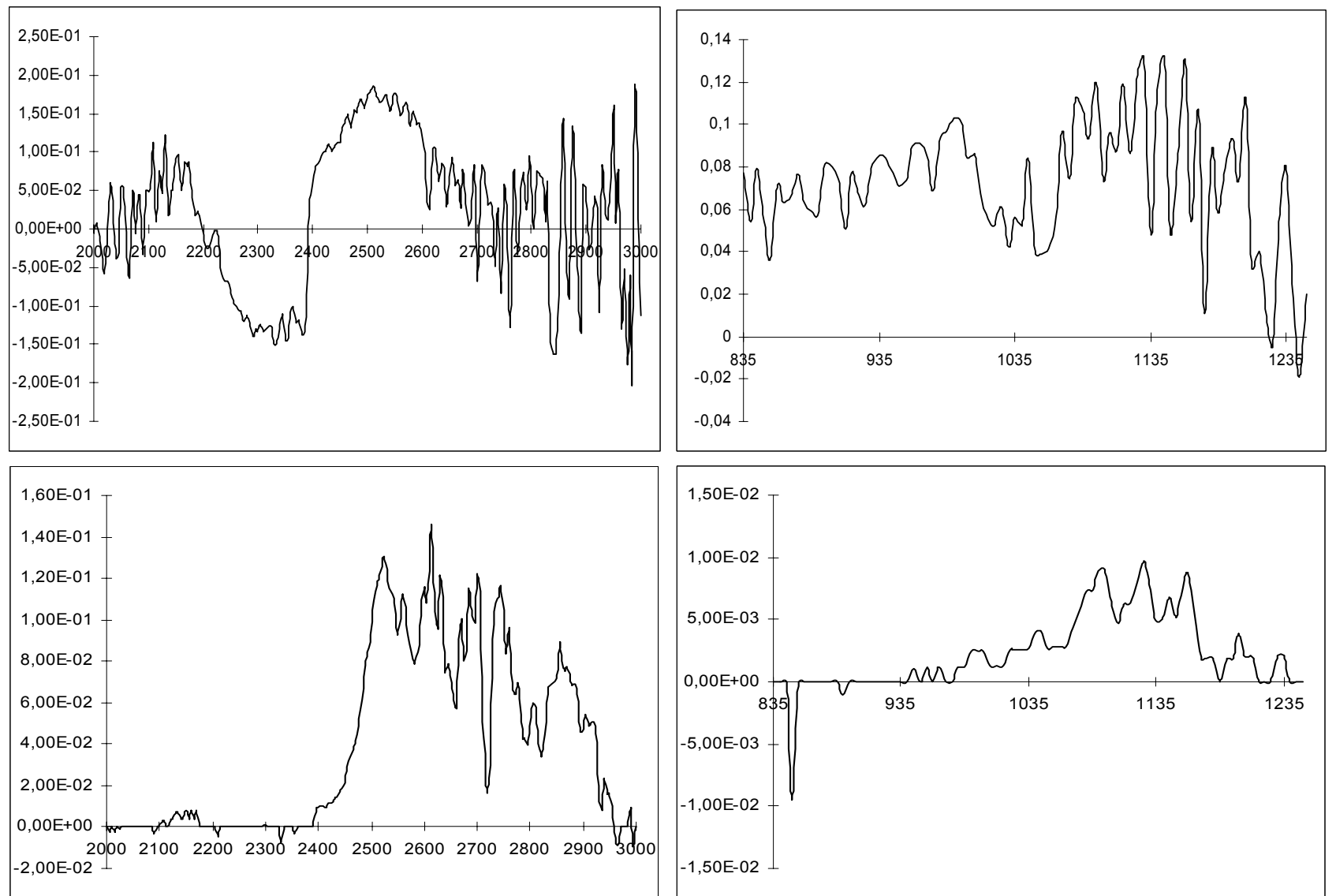

Figure 2: Relative accuracy of the MIBS background spectral modelling ( (model - reference) / model) in the MWIR (left) and LWIR (right) bands (x-axis is in $\left.\mathrm{cm}^{-1}\right)$. The top figures compare the spectral model with real recorded data (LAPTEX, $\left.T_{a i r}=24^{\circ} \mathrm{C}\right)$, the bottom figures compare the spectral model with the MODTRAN $\left(T_{\text {air }}=15^{\circ} \mathrm{C}\right.$ over $\left.39 \mathrm{~km}\right)$ reference path radiance. 
The scene regeneration process makes use of a profile database from which the appropriate profile is selected that closely fits the meteorological data for which the simulation is started. Primary selection choices are the wavelength band and air and sea temperatures (secondary: relative humidity, wind speed, wave height, solar irradiance). Profiles are then corrected for the actual band choice and air temperature. Spatial surface intensity structure and white caps are inserted as described in Schwering (1996) $)^{5}$. Since the start of the development additional effort have been taken to incorporate a background cloud model $^{6}$, as well as a spectral model and temporal model ${ }^{7}$. With this information better use could be made in cloudy scenarios, and for higher frame rate cameras with specific spectral characteristics (scenario or camera). These references also contain sets of validation parameters as well as an introduction of cloud structure in the scene generation and parameters describing levels and size of structured components. Typical sample cases have been prepared for cumulus, stratus type clouds.

The paths we are interested in for detection are long atmospheric paths. For these long paths the spectral components are dominated by the atmospheric path radiation. We have estimated this with black body radiation from the atmosphere at the local air temperature. We have assessed the validation by comparing this with real recorded infrared spectral data. Figure 2 shows the relative difference between background spectra near the horizon over sea. The data were taken from the NATO LAPTEX trials $\left(1996^{8}\right)$. In the MWIR band we calculate an average difference of $2.3 \pm 10 \%$ and in the LWIR band of $7.2 \pm 3 \%$. Hence the black body model predicts for both bands slight overestimates of the recorded broad band spectral data. Reasons for this can be mixed temperature contributions, good atmospheric transmission (in particular beyond $2400 \mathrm{~cm}^{-1}$ ). The fact that the range $2300-2400 \mathrm{~cm}^{-1}$, which is in fact identical to the local temperature due to the very low transmission caused by the $\mathrm{CO}_{2}$ absorption band at 4.3 micron, is underestimated shows that deviations of the local temperature compared to the average meteorological data accepted for the optical path. Also in the LWIR band expected deviations occur at the wave numbers with higher transmission values. Similar comparisons show deviations of our simple spectral model from MODTRAN spectral predictions (with a single air temperature) that are somewhat more severe in part of the MWIR band from 2500-3000 $\mathrm{cm}^{-1}$ leading to a broad band relative difference of $4.3 \pm 4.9 \%$ in the MWIR band and $0.2 \pm 0.3 \%$ in the LWIR band. From these data we have concluded that our purposes, the spectral data for long atmospheric paths can be well modeled by a black body component based on air temperature. This statement is supported by the fact that near horizon surface glints are largely reflected sky radiance over the measured longer paths. As the surface reflections can constitute of complex mixes of various spectral components, in the first order we have decided to scale the black body with the broad band intensity over the requested wavelength regime.

To incorporate temporal data at high frame update rates in the simulation, the relation between the scene time constant and the sampling rate becomes important. In Schwering $(1992)^{4}$ it was shown that for specific maritime scenarios, local temporal correlation occurs up to approximately $2 \mathrm{~s}$, and correlated motion can be distinguished somewhat longer. We therefore decided to start analyzing time constants in different scenes and also use the wave constants generated on the basis of the wind speed parameter. During regeneration, for each image location, random selections are drawn at the moments associated with the time constants, starting at the first image. Additionally separate phases are generated for each location as well, and intermediate generator values in between these time moments are interpolated with a cosine function. This assures that the intermediate values have the preferred kind of correlation. With these generators, the same spatial scene generation process is performed as presented in Schwering $1996^{5}$ for each of the images separately. In Figure 3 a sample regeneration process is given for a time series of a single image pixel, together with its (time-)spectral distribution. These sample data have been compared to wave height data from Konstantinov ${ }^{9}$. Although we are representing image intensities and the referred paper describes wave heights, a correlation between the two is expected. At present we assume that this shows evidence that the validation of the temporal data is assessed appropriately. 

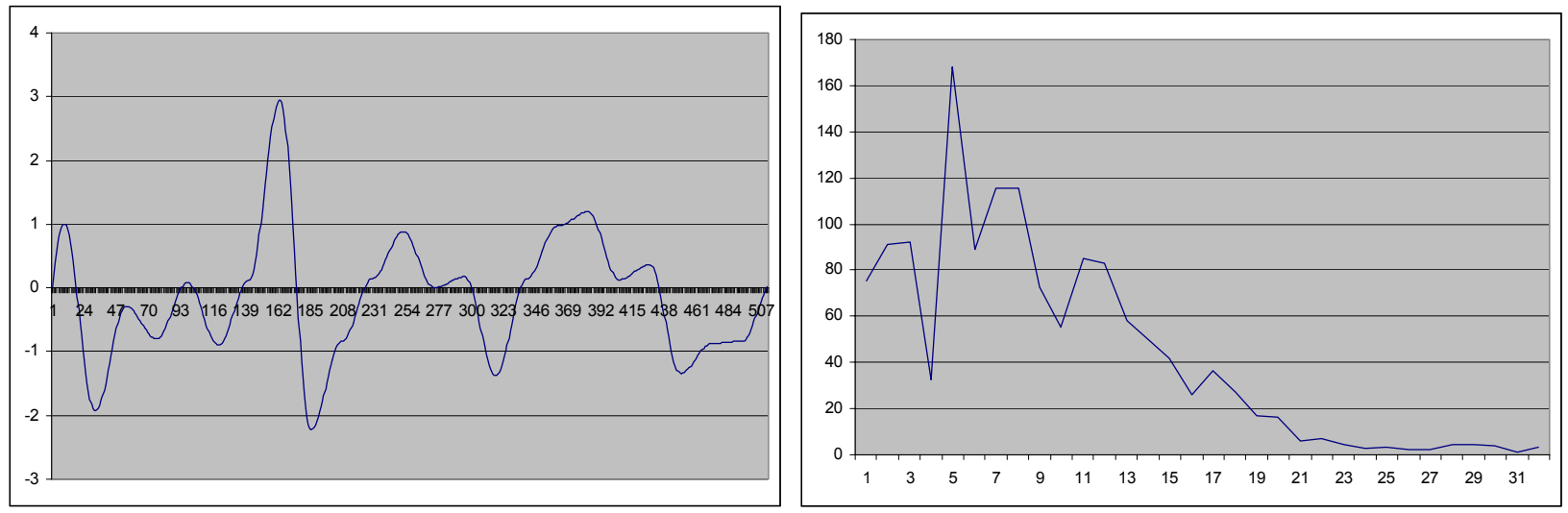

Figure 3: Harmonic noise generated time sequence of pixel intensity ( $x$-axis unit frame number) and its spectral distribution (x-axis unit 1/(512 frame numbers)). A time constant of 9 frame numbers was used in this simulation.

\section{ADDITIONAL DATA RECORDINGS}

In this section we describe some of the data recording that has been used to feed the modeling developments for the new operational scenarios. These data are important as they indicate clear requirements at a qualitative level. In order to balance the effectiveness of the model simulations, it is important to assess the various quantitative effects from the imagery. For example, particular background effects can be dominating the performance in cluttered conditions and can also dominate contrast values. In the present section we derive qualitative requirements for modeling efforts to support performance assessment for future IRST system development.

\subsection{Target measurements}

To assess the relevance of target signatures in different environments TNO has participated in trials at various locations around the world in cold environments (MAPTIP, 1993 $3^{10,11}$ ) Mediterranean environments (LAPTEX, 1996 ${ }^{8}$ ) and warm humid environments (WATIX, $1999^{12}$ ). The first two trials were performed under the guidance of the NATO/RTA research groups. The latter national trial took place in the Caribbean. During these trials, targets, background and atmospheric measurements were recorded for the assessment of detection and classification. In Figure 4 some long wave infrared (LWIR) examples are given of a small inshore craft at various distances from a sensor on shore $(0.3,0.6,1.0$, 1.5, 2.0 nautical mile (NM)). The figure also shows images of floating oil drums at distances of 0.5 and 1.0 NM. During the trials also data were recorded for other potential asymmetric targets such as Go-fasts, RHIB (Rubber Hull Inflatable Boat), and Jetskis. 


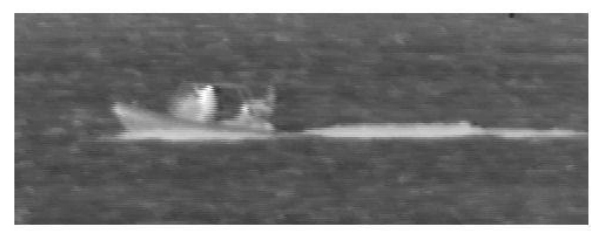

$0.3 \mathrm{NM}$

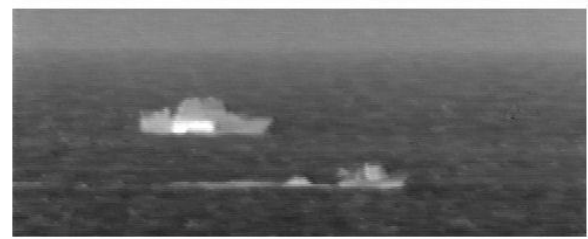

$0.6 \mathrm{NM}$

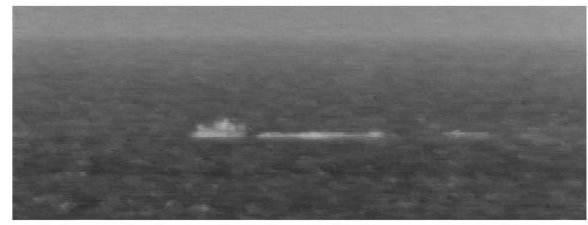

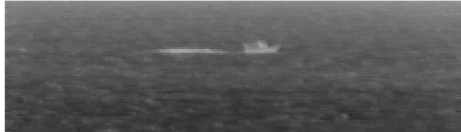

$1.5 \mathrm{NM}$

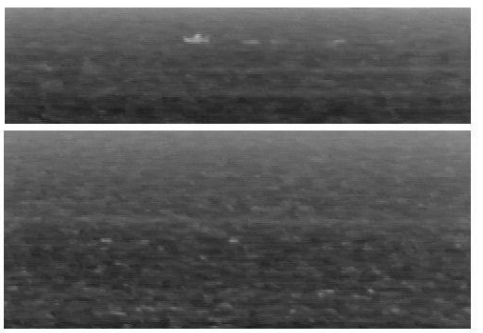

$2.0 \mathrm{NM}$

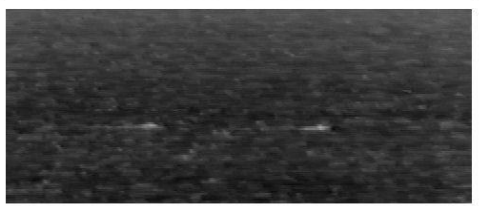

Drums

$1 \mathrm{NM}$

$1.0 \mathrm{NM}$

Figure 4: LWIR measurements of inshore craft at various ranges in a tropical environment (whiter grey values are warmer).

From the images in the figure it is clear that we can distinguish several features from the target. Due to the fact that short range targets are seen against a close-by sea background, the average apparent background temperature is lower than the sky (near horizon) temperature, and therefore the target has a larger average contrast difference with the local sea background. Intensity histograms of these small boats show several features such as hot (or better warm) spots, such as persons, engines and other warm areas that were heated up by the sun. Additionally, due to its speed, the produced wake can be significant. In size it can exceed the length of the boat by a factor of two and in integrated intensity it can exceed the total emission by the target itself. For smaller objects such as oil drums, detection becomes more complicated due to the temporal behavior of the wave structure. The wake effects are an indication that the interaction of the target with the environment should be taken into account. First of all for modeling purposes to better predict background (and obviously also target) intensity and clutter levels, but also in the detection process itself, as the wake can improve detection of moving targets it can also guide the sensor to the target through sophisticated classification algorithms.

\subsection{Background measurements}

Besides target signatures also background signatures are of great importance for the IRST detection process. There is a wide variety of potential background intensity levels and structures. The background influences the contrast that the target has in its local environment, and its structure has an influence on detection thresholds and false alarm rates. For system evaluation the absolute intensities are important with respect to spatial, temporal and spectral variations.

In Figure 5 some typical coastal backgrounds are presented in a cold and a warm environment. Typical coastal features as sand, rock and concrete buildings will be easily heated up by direct sunlight and will show a strong temperature increase as soon as it is heated by the sun. Other background structures, such as vegetation have a much slower increase in temperature (or none at all) and therefore show a weak diurnal variation. In particular in coastal environments a strong intensity structure influence and accompanying variations are expected that can show up as strong clutter sources. In Figure 5 the cold environment image only shows some manmade structures on the beach side and activity (cars) in a almost clutterless environment, while the image recorded in the warm environment clearly shows strong infrared emission from the heated up rock. It is clear that this will decrease the target contrast for any target in front of this type of background. Note that this clearly exceeds the background levels of both sea and horizon air intensity levels. 

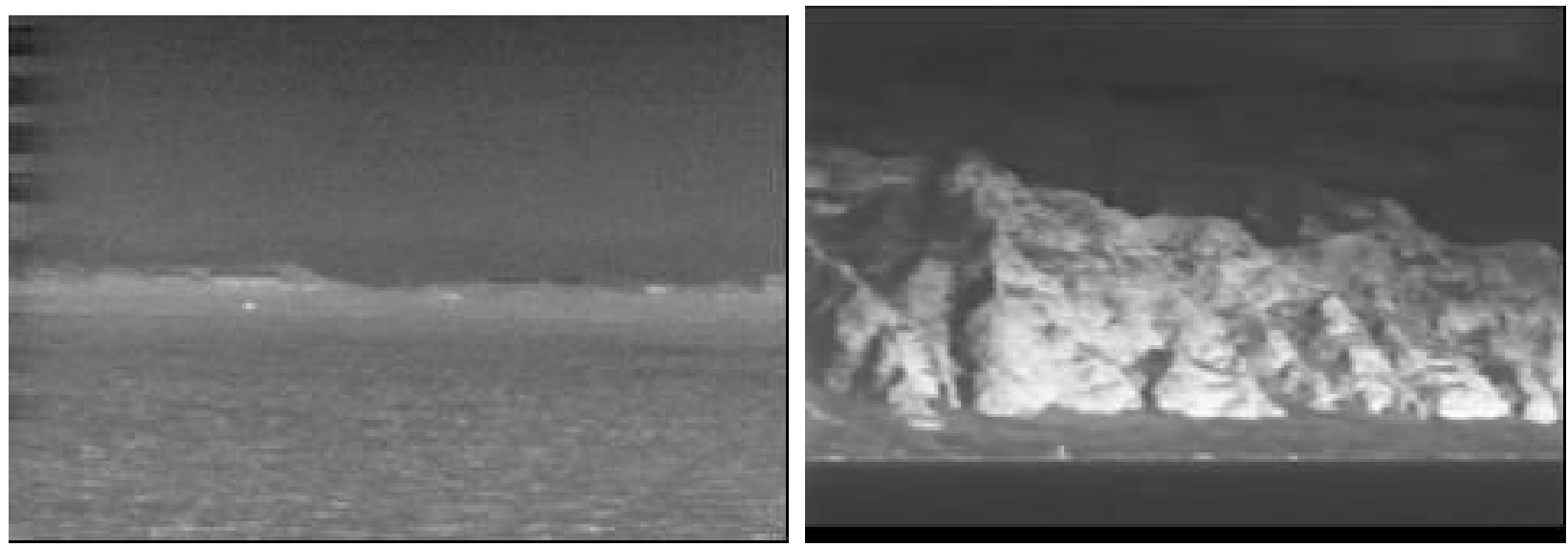

Figure 5: LWIR measurements of a cold environment Hook of Holland and a warm environment in Hawaii (whiter grey values are warmer).

Of great importance for detection and classification are the spatial scales and temporal scales of the background structure. At open sea clouds and cloud structure are relevant and the typical horizon jumps (Schwering, $1996^{5}$ ). Furthermore the sea surface intensity level is dominated by a background level originating from both the sea temperature as well as the reflecting sky radiance at grazing angles. The structure of the sea surface in the infrared is generated by waves and white caps and follows a random behavior. Typical spatial and temporal effects in the sea background can lead to strong clutter responses and shadowing of objects behind the waves. For long horizontal paths in warm environments the LWIR bands do not show a clear horizon jump. This is due to the poor transmission in humid environments in this band.

Figure 6 shows additional clutter complications in coastal environments. Beside the presence of layered clouds, that may be hampering detection of low observable air targets, the background level of the coastal land background is strong, and may limit contrasts of targets severely. Furthermore the presence of birds may result in false alarms in the MWIR and LWIR bands. Strong contrast values are recorded on seagulls at relatively short ranges (such as the one seen in this figure). Other coastal effects of direct importance are the coastal sun glint contributions.

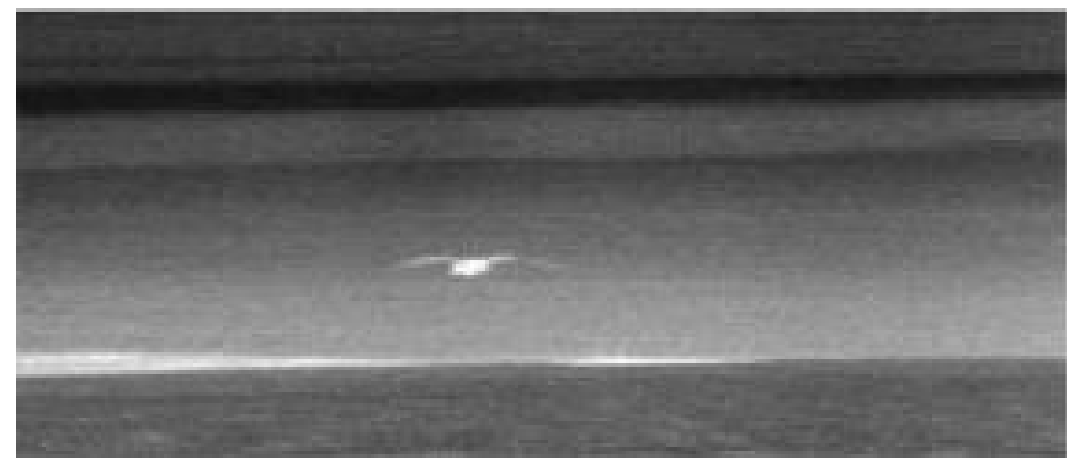

Figure 6: LWIR measurements of an inbound seagull, layered cloud structure and warm islands at the horizon in the Baltic Sea (May, 1999). 
Figure 7 shows temporal intensity fluctuations in the mid infrared wavelength band (MWIR) during trials in the Baltic in 1999. The images were recorded over sea in a field with clear sky. From the figure it is obvious that the intensity fluctuations are stronger in amplitude near the horizon than at higher elevations. The fluctuations also show a longer period near the horizon than at higher elevations.

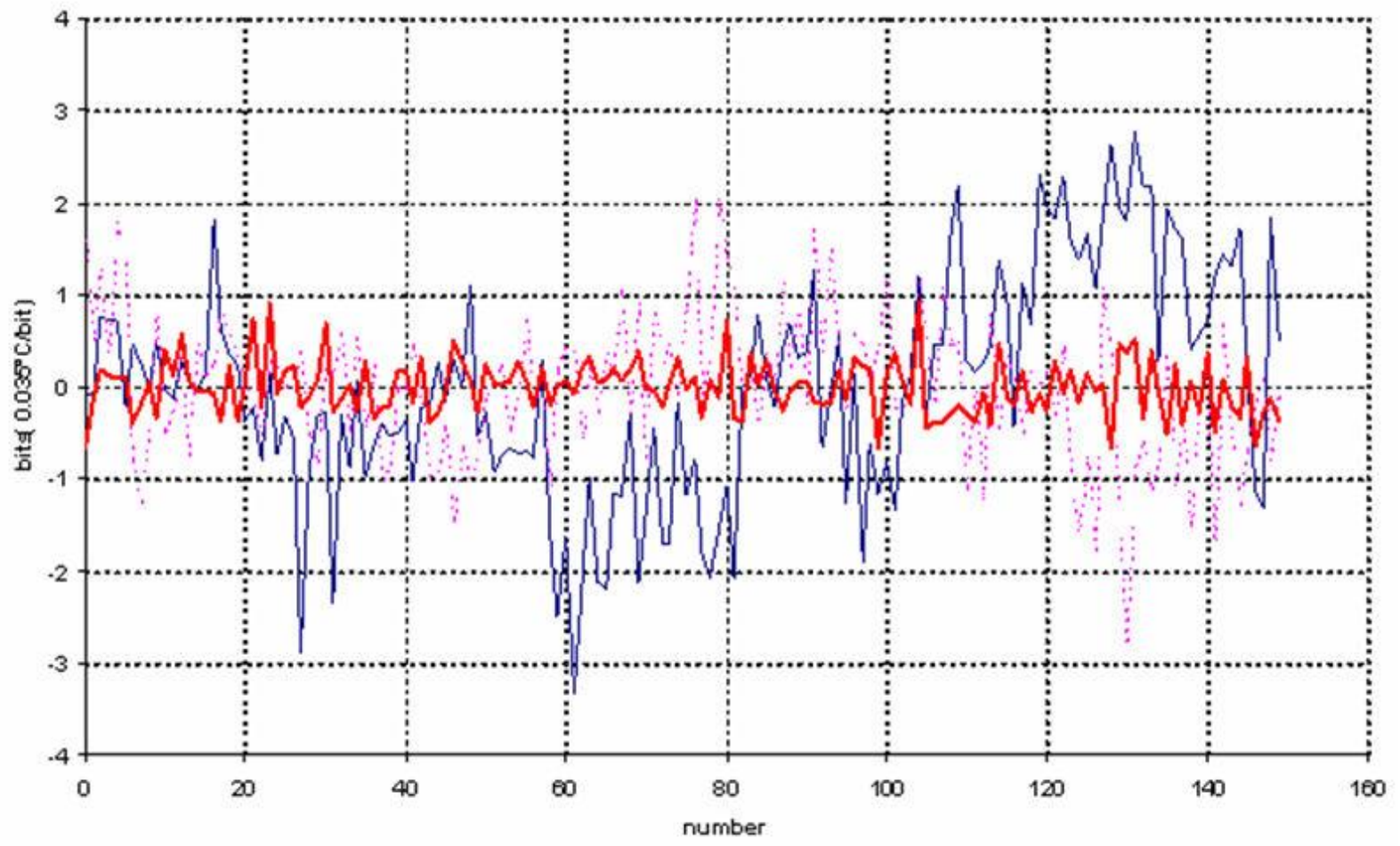

Figure 7: MWIR temporal intensity fluctuations of an air pixel (in arbitrary units) in successive image frames (6 seconds at $25 \mathrm{~Hz}$ ) at the horizon (red solid near zero), somewhat above the horizon (dotted purple), and at high elevation in the image (blue thick solid). Data recorded in the Baltic (May, 1999) with mean value set to zero.

\section{MODEL COMPONENT DEVELOPMENTS}

In the paper we discuss methodologies to incorporate the effects of environments and scenarios in the testing of IRST systems. One of the main drivers for detection of dim targets is the background intensity and clutter levels. The proposed methodology is based on experience with sea based IRST trials combining the possibilities of performance assessment in required scenarios to the real performance in available coastal scenarios. From the data derived in the previous section we have determined a set of requirements for the scene modeling that supports the analysis and performance assessment for the future IRST. These additional mathematical modeling should find its primary basis in physics formalisms. Primary areas of research are the coastal backgrounds (intensity and clutter), target-surface interfacing (such as wakes), as well as introduction of clutter objects (e.g. birds) in the same sense as targets can be introduced in simulations.

\subsection{System and model integration requirements}

In general scenario modeling is supported with system modeling. For accurate system testing, the simulation model should be better in resolution and dynamic range than the system under test. From the system point of view simultaneous 
infrared bands are assumed. At present a system resolution of $0.1 \mathrm{mrad}$ at $25 \mathrm{~Hz}$ seems applicable. It is anticipated that other (visible light) bands can become important at short ranges.

For this purpose testing procedures depend strongly on accurate infrared target, background and atmosphere models. In particular background effects can be dominating for performance in clutter conditions and can also dominate contrast values. A requirement of the scenario model should be that it can be easily integrated with a system model. The system model should contain both sensor and processing modules. The scenario and system models should work at the same level of detail of the spatial, temporal and spectral domain. An example of such an integrated model is IRSCENE ${ }^{2}$ within the EOSTAR model suite ${ }^{3}$. Further integration in system models is basically done at image level. The latter can be performed for validation of these sensor models.

When targets are inserted in the backgrounds, target and background pixels are mixed. For future threats also the interaction of the target and the sea surface needs to be taken into account. Fast surface vessels, such as a Jetski, can move at speeds reaching $20 \mathrm{kts}$ and higher. Even at relatively slow speeds a wake is produced. This foam will contribute to the signature. First analysis show that during beam on aspects angles, the visible length of targets can increase easily by a factor of two (see Figure 4) due to the length on the wake.

\subsection{Maritime background model developments}

The Maritime Infrared Background Simulator (MIBS) was developed for analysis of airborne targets in a naval environment. From the model description as presented before, several approaches were followed to expand into the new warfare areas. At present, the model generates scene image sequences containing background structure, including sea, sky, clouds, coastal and sun glint information up to a frame rate of $25 \mathrm{~Hz}$.

With present-day target modeling accurate values for the vessel surface temperature can be determined from the presence of internal heat sources, solar heating, wind cooling, and reflection effects. For background imagery the physics based modeling needs to be supported by scene simulations based on random processes. We do not expect to know the surface structure in sufficient detail to understand the entire sea surface. The same is true for the clouds structure. However we still want to know accurate background intensity levels and clutter indicators.

A true complication of the evaluation models will be the assessment of the interaction between targets and backgrounds. It is clear from the previous sections that the targets have an impact on the intensity levels near the target. The levels are influenced mainly by the turbulence caused at the water level and spray. The interaction of the wake depends on several quantities. The presence of a target and its orientation is the primary factor. Then we have to take into account the surface structure present in the open sea. This is based on the same parameters that determine waves, such as sea depth, wind speed etc. A target that moves in the water will interact more strongly when the speed is higher and possibly when it moves in a certain direction (perpendicular to) with respect to the dominant waves. Then the effects of special sources, such as the sun, can cause strong reflection in specific looking angles. This also depends on the sensor location. Furthermore the V-wake shape can be visible when looking at (nearly) inbound or outbound targets, while for passing targets a long tail wake appears present. These effects are pure disturbances of the surface structure and are based on reflection differences. Additionally some real temperature effects may occur when the targets exhaust mixes with water, and especially with mixing colder or warmer water layers from below the surface. From the temporal fluctuations in real measurements up to now, the dominating effect is related to variations in reflection.

In Figure 8 some generated images are presented with sun glints and with warm coastal backgrounds. Both images are generated with cloud background inserted, and show realistic features. For coastal backgrounds a comparison of this figure with Figure 5 and Figure 6 shows the effectiveness of the horizon modeling process. In Figure 9 we have inserted some EOSM (Electro Optical Signature Model, Neele $2005^{13}$ ) target simulations (RHIB and a small tug boat) inside the generated MIBS backgrounds. At present no wake is present in the scene generation, showing the need for accurate wake modeling. When compared to Figure 4 additional wake modeling will lead to larger sized target signatures with specific spatial and temporal features and possibly more intense radiation.

In generation of coastal backgrounds a number of horizontal surface layers are introduced at different apparent temperatures. Coastal contours are modeled as fractal noise patterns at positions based on the horizon position. Intensity 
levels are described as fractions of the horizon air intensity levels. The sea surface area affected by sun glint is determined by the solar diameter and the wind speed as it ruffles the sea surface and has an impact of the surface reflectance. Also an effective smoothing near the horizon is taken into account in the sun glint appearance.

Although the first results look promising, accurate intensity and structure validation still needs to be performed. Furthermore the presence of persons and other small hotspots on small surface targets is important for detection and classification.
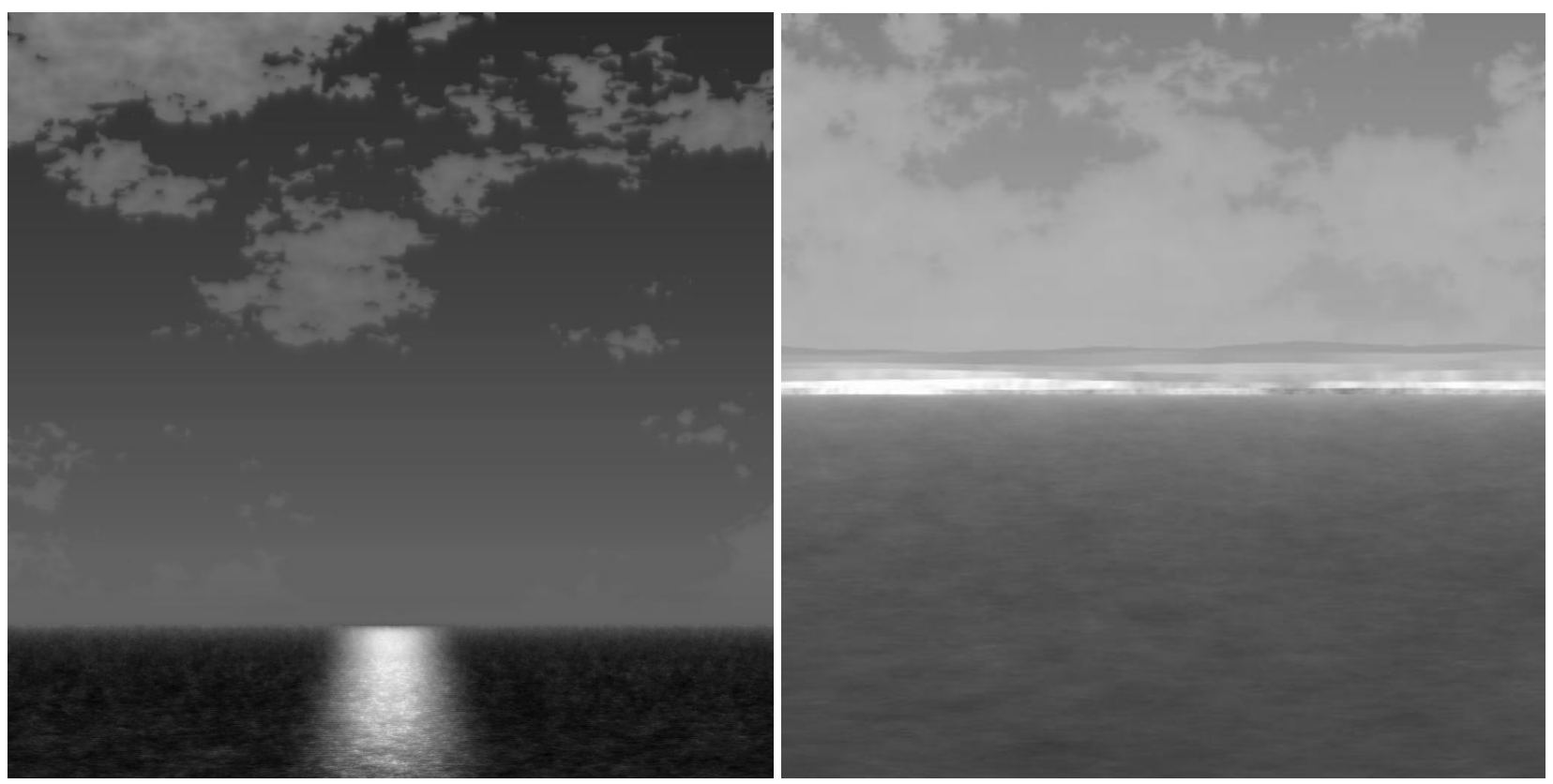

Figure 8: Maritime cloudy MWIR simulations of sun glints (left) and coastal (right) backgrounds. (In the image regeneration process of sun glints, direct sunlight and scattered skylight are not yet incorporated).
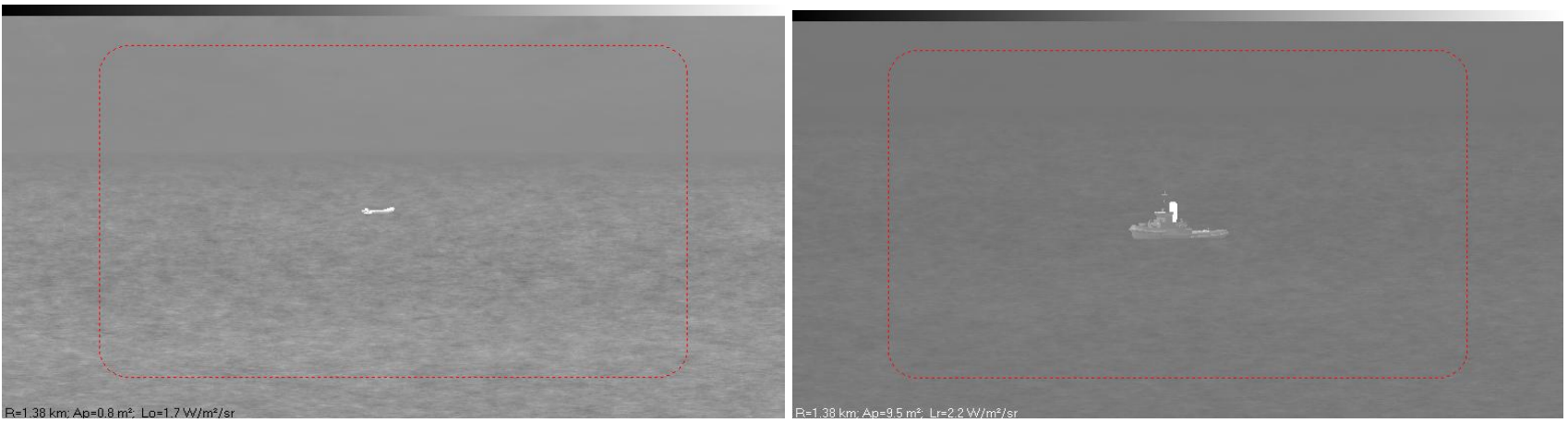

Figure 9: Simulated MWIR RHIB (left) and small tug boat (right; both generated by EOSM) and integrated in MIBS inside EOSTAR. 
Until now validation of MIBS background modeling has been performed for the background intensity with respect to

- $\quad$ sea surface, white caps, and clouds

- $\quad$ spatial up to $0.1 \mathrm{mrad}$

- $\quad$ spectral in MWIR and LWIR bands spectrally resolved

- temporal variation from 1 to $25 \mathrm{~Hz}$

\section{DISCUSSIONS}

The present status of the MIBS model is a next step into accurate background prediction required for system performance assessment. For future developments the primary aspects that will be tackled are based on the analysis of critical features. Although they may change in time, depending on future IRST system concepts and threats, at present the following model development subjects are foreseen.

For improved use in target detection algorithms, introduction of target surface interface information such as wake and spray generation is required. The potential interaction with background is also important. Targets need to be incorporated with appropriate smoothing into the background to avoid sharp target edges in the images. For air targets this was performed in IRSCENE, based on target sub-pixel filling factors. EOSTAR already performs similar integration for surface targets.

To assess the effects of surface clutter generated by white caps, an introduction of this topic based on white cap distribution spectra is required in the modeling. This spectrum is based on wind speed and history parameters. Also in that case the long tail histogram should be accompanied by continuity in the sea surface intensities.

Additional clutter sources should be introduced, such as birds and flock of birds. These can be incorporated in similar ways as targets are inserted in infrared imagery. Other sources of false alarms might be of relevance in the future as well. As for targets, accurate insertion techniques are required, providing a smooth intensity overlap of object in the backgrounds.

The present version of MIBS is a stand alone version (C-code and Java shell). Integrated applications are available in the IRSCENE and EOSTAR image generators in maritime scenarios. Beside further model developments, validation of all model aspects will continue in different scenarios. In particular validation of temporal effects, sun glint and coastal backgrounds is anticipated, as well as newly developed features.

\section{CONCLUSIONS}

In this paper we have described the Maritime Infrared Background Simulator (MIBS). The model was developed for IRST performance assessment, and based on sea surface structure analysis with the aim of background predictions near the horizon. A description is given of the spatial, temporal and spectral aspects of the modeling components, as well as validation efforts in these fields. Furthermore additional model features are described that include assessment of systems in coastal environments and environments with specific backgrounds such as clouds and sun glints. As the model is integrated in scene generators, such as IRSCENE and EOSTAR, integration topics related to the insertion of targets and the interaction of targets with their local background, are discussed. Validation work on spatial, temporal and spectral intensity information for the model, are described. New developments will take place in the sea surface interactions between targets and backgrounds and validation aspects in coastal scenarios. 


\section{ACKNOWLEDGEMENTS}

This work for this paper was supported by the Netherlands MoD under the program V509. Although this work is supported by the MoD, it might or might not reflect the position of the MoD or the NL government. Spectral data recorded at LAPTEX was made available by the Canadian DRDC. Dr. F.P. Neele is acknowledged for generating specific EOSM and EOSTAR simulations. In the analysis of the modeling efforts, data from various NATO trials was used.

\section{REFERENCES}

1. $\quad$ Knepper, R., Sirius, a long range infrared search and track system, SPIE Proc. Vol. 3061, 578-584, 1997.

2. $\quad$ Schwering, P.B.W., de Jongh, E.J.C., IRSCENE User Manual, TNO, 26054/DF001, November 2002.

3. Kunz, G.K., M.A.C. Degache, M.M. Moerman, A.M.J. van Eijk, F.P. Neele, S.M. Doss-Hammel and D. Tsintikidis, Status and developments in EOSTAR, a model to predict IR sensor performance in the marine environment, SPIE's 11th International Symposium on Remote Sensing, 'Optics in Atmospheric Propagation and Adaptive Systems VII', Maspalomas, Gran Canaria, September 2004.

4. Schwering, P.B.W., Infrared clutter measurements of marine backgrounds, SPIE Proc. Vol. 1486, 25-36, 1991.

5. $\quad$ Schwering, P.B.W., Maritime infrared background clutter, SPIE Proc. Vol. 2742, 255-266, 1996.

6. de Jongh, E.J.C., Schwering, P.B.W., MIBS Feasibility Study Cloud Layer Insertion, 31425/AM001, July 2001.

7. Schwering, P.B.W., de Jongh, E.J.C., Maritime Infrared Background Simulator (version 2), TNO report FEL98-A046, January 1999.

8. de Jong, A.N., Winkel. J., Point target extinction and scintillation as function of range at LAPTEX Crete, SPIE Proc. Vol. 3125, 167-179, 1998

9. Konstantinov, O., Measurement of sea level, wave height and slopes by analysis of sea surface image sequence from polarization camera, Pacific Oceanological Institute, Vladivostok Russia.

10. McGrath, C.P., Schwering, P.B.W., Fritz, P.J., Comparison of MAPTIP FLIR detection ranges to the EOTDA prediction model, SPIE Proc. Vol. 2828, 76-84, 1996.

11. Schwering, P.B.W., de Leeuw, G., van Eijk, A.M.J., Transmission of 10 micron radiation over coastal waters: comparison of point source image intensities with aerosol extinction and MODTRAN calculations, SPIE Proc. Vol. 2828, 64-75, 1996.

12. Schwering, P.B.W., de Jong, A.N., Winkel, J., Kemp, R.A.W., Resultaten van het Warm-humid Thermal Imager Experiment WATIX, TNO report FEL-00-A116, The Hague The Netherlands, 2000

13. Neele, F.P., Infrared ship signature prediction, model validation and sky radiance, SPIE Proc. Vol. 5811-24, 2005. 